\title{
Uma Experiência de Avaliação Multidimensional de Cursos de Redes de Computadores em Ambientes de Testbeds
}

\author{
Jane B. Santos ${ }^{1}$, Adriana V. Ribeiro ${ }^{1}$, Italo Valcy ${ }^{1}$, Maria C. S. de Souza ${ }^{2}$, \\ Ecivaldo S. Matos $^{1}$, Leobino N. Sampaio ${ }^{1}$ \\ ${ }^{1}$ Departamento de Ciência da Computação \\ Instituto de Matemática e Estatística - Universidade Federal da Bahia (UFBA) \\ Salvador - BA - Brasil \\ ${ }^{2}$ Departamento de Administração - EAUFBA \\ Programa de Pós-graduação em Gestão Social - CIAGS/UFBA \\ \{jane.santos, adrianavr, italovalcy, mcarols, ecivaldo, leobino\}@ufba.br
}

\begin{abstract}
Testbed environments are well-used for research involving Computer Networks. However, few studies explore its potential for supporting teaching activities. Furthermore, there is no system to guide the teacher in the use of these environments to enhance student learning, neither evaluation techniques that consider their particularities. In this article, we introduce the results of the application of an evaluation RUBIK created to support the teacher in the preparation and validation of courses using testbeds. The results showed that our approach helps the teacher by identifying strengths and weaknesses, in addition to promoting student-centered learning.
\end{abstract}

Resumo. As redes de experimentação (testbeds) são comumente utilizadas para realização de pesquisas em redes de computadores, entretanto, poucos trabalhos exploram seu uso como ferramenta de apoio às práticas de ensino. Além disso, não há um sistema que oriente o professor no uso desses ambientes para potencializar a aprendizagem pelos alunos, tampouco há técnicas de avaliação que considerem suas especificidades. Neste artigo, apresentamos os resultados da aplicação do dispositivo de avaliação RUBIK criado para apoiar o professor na elaboração e validação de cursos utilizando testbeds. Os resultados demonstraram que o dispositivo auxilia o professor através da identificação de pontos fortes e fracos, além de promover uma aprendizagem centrada no aluno.

\section{Introdução}

Nos últimos anos, diferentes iniciativas de construção de plataformas de experimentação em redes de computadores (testbeds) têm sido realizadas no Brasil, apoiadas, em sua maioria, pela Rede Nacional de Ensino e Pesquisa (RNP) [Dias et al. 2019]. Tais ambientes são caracterizados pela disponibilização de recursos computacionais compartilhados, distribuídos, com acesso remoto e, muitas vezes, em larga escala [Tavares 2018]. Inicialmente, os testbeds foram utilizados predominantemente para fins de pesquisa [Berman et al. 2014]. A partir da consolidação e popularização das tecnologias utilizadas, tais ambientes passaram a beneficiar um conjunto mais amplo de atividades, que inclui as ações de ensino de redes. Entre as iniciativas lideradas pela RNP, destaca-se a 
IX Congresso Brasileiro de Informática na Educação (CBIE 2020)

Anais do XXXI Simpósio Brasileiro de Informática na Educação (SBIE 2020)

construção do testbed FIBRE (Future Internet Brazilian Environment for Experimentation) [Ciuffo et al. 2016], uma infraestrutura de pesquisa focalizada na experimentação e aberta para a utilização por estudantes e pesquisadores brasileiros.

Atualmente, o FIBRE funciona como uma espécie de laboratório virtual que permite aos alunos desenvolverem, aprofundarem e validarem experimentos em redes de computadores em uma fatia real da rede [Ciuffo et al. 2016]. Assim, um dos principais benefícios está no fato de os experimentos serem realizados em equipamentos reais, similares àqueles utilizados nas redes de produção, que não estão disponíveis em laboratórios tradicionais. Tais benefícios levaram a RNP a lançar, em 2015, uma chamada de projetos de elaboração de materiais didáticos para ambientes de testbed ${ }^{1}$, no intuito de incentivar o uso do FIBRE em sala de aula. Através desta iniciativa, buscou-se oferecer um ambiente de experimentação para práticas de ensino de redes a alunos e professores.

$\mathrm{O}$ uso de ambientes de testbed no apoio ao ensino e à aprendizagem de redes de computadores demanda, em primeira instância, a elaboração de materiais didáticos para apoio ao professor e ao aluno. Considerando que o uso de testbed no ensino é algo recente, observa-se carência de instrumentos de avaliação dos cursos ofertados em consonância com os materiais didáticos disponibilizados. As avaliações existentes se resumem à adoção de mecanismos de ensino, que consistem na coleta de dados por meio de questionários aplicados ao final dos cursos. Apesar de ser possível obter algumas informações sobre a experiência do aluno no curso, essa forma de avaliação desconsidera outras dimensões de análise.

Diante da carência de dispositivos de análise, este trabalho apresenta um relato de experiência de avaliação de um curso de extensão em testbed. A avaliação fez uso do dispositivo RUBIK que permite a análise de múltiplas dimensões. Essa experiência auxilia na sinalização de critérios importantes a serem considerados para o planejamento de cursos dessa categoria, assim como apoia a verificação do atendimento desses critérios durante e após a conclusão do curso.

As demais seções do artigo estão organizadas da seguinte forma. Na Seção 2 apresentamos alguns trabalhos relacionados sobre o uso de testbeds no ensino de redes. Na seção 3 são discutidas algumas metodologias de avaliação e apresentado o dispositivo de avaliação RUBIK. Na Seção 4 é detalhada a experiência do curso de extensão com uso de testbed. A Seção 5 apresenta os resultados da avaliação do curso por meio do dispositivo RUBIK. Por fim, a Seção 6 apresenta as considerações finais do trabalho.

\section{Iniciativas de Ensino em Ambientes de Redes Experimentais}

$\mathrm{O}$ uso de testbeds em espaços educacionais tem se mostrado uma alternativa promissora para o ensino de redes de computadores. Sobretudo porque os recursos computacionais disponíveis compõem laboratórios capazes de oferecer uma prática diferenciada aos alunos [Mikroyannidis et al. 2017, Pedroso et al. 2018, Valenzuela and Amaral 2018].

[Mikroyannidis et al. 2017] demonstrou através da iniciativa Forging Online Education through FIRE (FORGE) que testbeds podem impactar positivamente professores e alunos na área de redes de computadores, já que permitem aos alunos desenvolver autonomia em sua aprendizagem, modificando assim a relação aluno-professor-aluno. A

\footnotetext{
${ }^{1}$ https://www.fibre.org.br/documentation/courseware/
} 
avaliação desse curso foi realizada pelos alunos através do preenchimento de um questionário. Os resultados demonstraram que a realização de experiências práticas no testbed FIRE os ajudou na compreensão dos conceitos teóricos. Por sua vez, [Pedroso et al. 2018] utilizaram o testbed FIBRE para compor um protótipo que propõe experimentos capazes de auxiliar na pesquisa e no ensino de redes de computadores, proporcionando a alunos e pesquisadores um ambiente flexível e de fácil provisionamento. Apesar de não realizar cursos para experimentar o impacto do protótipo na aprendizagem dos alunos, os autores detalham trechos de experimentos que podem ser facilmente replicáveis por professores. Já em [Valenzuela and Amaral 2018], os autores descreveram a implementação de um laboratório educacional de roteamento dinâmico desenvolvido, por meio da infraestrutura do testbed FIBRE, para oferecer a professores e alunos de redes de computadores um ambiente de testes mais próximo da realidade. Apesar de não terem utilizado o laboratório em termos práticos, os autores afirmam que através do sistema desenvolvido é possível criar topologias inteiras para o ensino de redes de forma mais próxima da realidade.

Apesar de esses trabalhos incentivarem e demonstrarem o uso de testbeds com foco educacional, nem todos realizaram cursos para validar os experimentos com alunos, e o que realizou não apresenta um sistema de avaliação capaz de garantir sua efetividade no ensino, nem na aprendizagem de redes de computadores. Portanto, para avaliar a experiência relatada neste trabalho foi necessário o desenvolvimento de um dispositivo de avaliação denominado RUBIK, que se encontra descrito na próxima seção.

\section{Metodologias de Avaliação}

Para definir um dispositivo de avaliação, foi preciso eleger indicadores que servissem de suporte à avaliação. No contexto de testbeds é importante que esses indicadores considerem as especificidades do ambiente, principalmente no que tange a postura docente para promover e orientar exercícios práticos nessas infraestruturas. Atualmente, alguns estudos [Silva and Silva 2008, Khan 2001] contribuem na seleção de indicadores de avaliação.

[Khan 2001] desenvolveu um framework de educação a distância (EaD), a partir da premissa de que as pessoas estão tão acostumadas com um modelo de ensino no qual professores e alunos estão no mesmo espaço físico, que acabam não se dando conta das inúmeras possibilidades geradas pela modalidade $\mathrm{EaD}$. O framework é composto por oito dimensões: Pedagógica, Tecnológica, Design de Interface, Avaliação, Apoio aos Recursos, Ética, Gestão e Institucional, e costuma ser utilizado para avaliação de experiências em EaD. Já [Silva and Silva 2008] apresentaram uma metodologia que contempla os quatro eixos canônicos do modelo de avaliação: o quê, quando, quem e como avaliar. Esses eixos integram desde os atores envolvidos na avaliação até os objetos avaliados.

Ao observar a relação entre os quatro eixos canônicos de avaliação considerados por [Silva and Silva 2008] e as dimensões expostas no framework de [Khan 2001], bem como os principais requisitos que devem ser avaliados em um curso utilizando testbeds como plataforma de ensino (criatividade, dinamismo, interação, inovação, etc.) foi possível desenvolver um dispositivo de avaliação composto por dimensões e indicadores para esse contexto, conforme detalhado na subseção seguinte.

A escolha por essas 2 (duas) teorias para embasar a construção do RUBIK deveuse ao fato de que, tanto [Khan 2001] quanto [Silva and Silva 2008] propõe estratégias que permitem analisar e avaliar aspectos considerados essenciais para garantia da quali- 
dade de cursos na modalidade EaD e/ou semipresencial, como: os participantes, práticas pedagógicas, softwares, hardwares, entre outros. Como a avaliação de cursos utilizando testbeds como ambiente de laboratório não é algo trivial, optou-se pela junção desses métodos de forma a garantir que todos os aspectos fundamentais expostos por [Khan 2001, Silva and Silva 2008] pudessem ser devidamente avaliados.

\subsection{Dispositivo de Avaliação RUBIK}

Para avaliar o curso sob diferentes perspectivas, foi criado o dispositivo de avaliação RU$\mathrm{BIK}^{2}$. O objetivo do RUBIK é avaliar a efetividades de testbeds no ensino de redes, bem como se tornar uma ferramenta genérica para avaliação de cursos que utilizam testbeds como infraestrutura tecnológica para a realização de laboratórios práticos. Apesar de Khan considerar 8 (oito) dimensões separadamente, na construção do RUBIK optou-se pela adoção de apenas 5 (cinco), onde as dimensões de gestão e tecnológica foram agrupadas em uma só (por avaliar aspectos semelhantes), e as dimensões institucional e de apoio aos recursos foram excluídas (por não haver dados suficientes para sua análise).

Assim, o RUBIK é composto por 5 (cinco) dimensões, consideradas essenciais para uma experiência de ensino: pedagógica, tecnológica e de gestão, design de interface, ética e avaliação. Para cada uma dessas dimensões foram considerados indicadores capazes de mensurar o impacto de cursos com uso de testbed em relação a diferentes aspectos, como uso de recursos tecnológicos, participação dos alunos, produção de conteúdo, etc.

As dimensões definidas no dispositivo RUBIK e seus respectivos indicadores podem ser observadas na Figura 1. Enquanto alguns indicadores foram definidos pelos autores com base na observação do curso, outros se basearam na discussão apresentada na literatura por [Crivelaro 2014], que tem foco em processos colaborativos. Essa escolha deveu-se ao fato de a necessidade de colaboração entre seus usuários na resolução de problemas complexos ser inerente aos ambientes de testbeds.

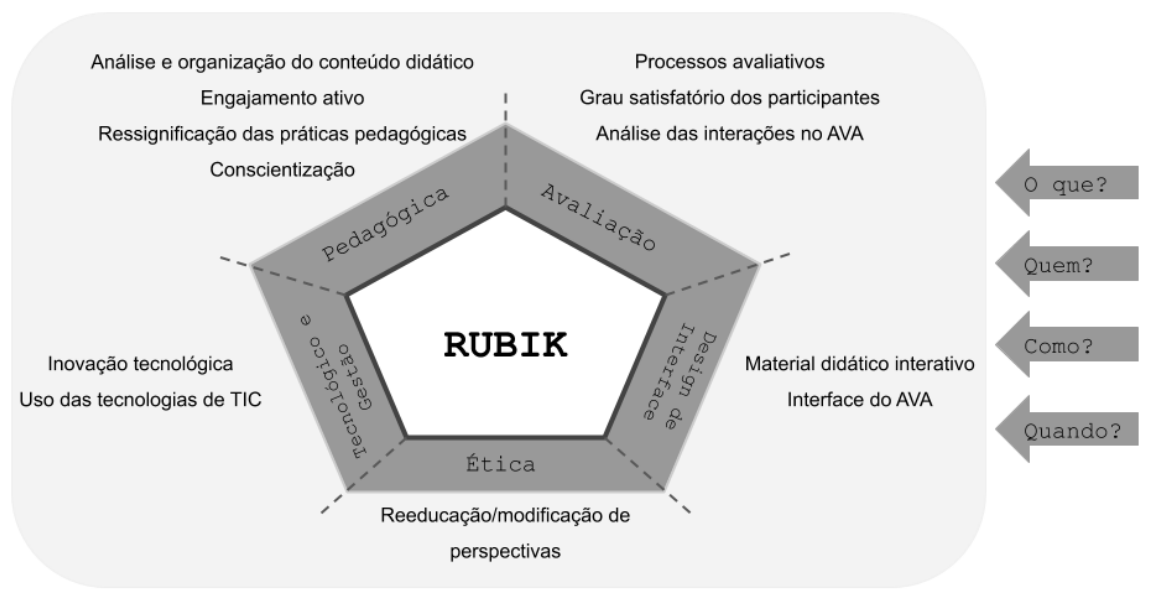

Figura 1. Dispositivo de avaliação RUBIK

Para cada um dos indicadores destacados que se relaciona com as dimensões especificadas na avaliação, alguns descritores também foram associados. Os descritores

\footnotetext{
${ }^{2}$ O nome RUBIK faz alusão ao quebra cabeça tridimensional conhecido como "cubo de Rubik" ou "cubo mágico", desenvolvido pelo húngaro Ernõ Rubik e que deve ser resolvido sob a perspectiva de múltiplas dimensões. https://www.rubiks.com/en-us/about
} 
representam o eixo "O que avaliar?" de [Silva and Silva 2008] e determinam o que foi avaliado em cada indicador. Os demais eixos foram representados da seguinte forma:

- "Quem avalia": o curso foi avaliado pelos professores, pelos alunos e autores deste trabalho através do dispositivo de avaliação RUBIK.

- "Como avaliar": análise do ambiente virtual de aprendizagem (AVA), do material didático, dos questionários aplicados aos alunos e entrevistas com professores.

- "Quando avaliar": antes do início do curso foi realizada uma avaliação para verificação do nível de conhecimento da turma; no entanto, a avaliação do curso, propriamente dita, ocorreu ao final de sua realização.

\subsection{Dimensão/Indicador}

Cada dimensão do RUBIK é formada por indicadores que possuem diversos descritores associados a eles. Neste trabalho, alguns descritores são de autoria própria, enquanto outros foram extraídos do trabalho de [Crivelaro 2014] e contextualizados dentro do cenário de uso de testbeds para ensino de redes de computadores, com a finalidade de fornecer aos professores um ponto de partida para o planejamento do curso, assim como, recursos que possibilitem a avaliação do curso após o seu encerramento.

A dimensão Pedagógica é responsável pelos aspectos relacionados ao ensino e aprendizagem como forma de promover uma mudança significativa na organização da sala de aula. Os descritores dessa dimensão analisam aspectos desde a seleção de conteúdos didáticos, proposição de metodologias inovadoras, estímulo do convívio social até a aprendizagem efetiva e colaborativa. A dimensão Ética, por sua vez, é responsável por analisar a relação dos alunos com os professores, além da postura dos professores no decorrer do curso. O objetivo dos seus descritores é verificar e avaliar as formas de interação e colaboração para criar um ambiente de ensino que facilite a aprendizagem. Já a dimensão Tecnológica e de Gestão é responsável pela definição das tecnologias a serem utilizadas no curso, identificando como elas contribuem para proporcionar um ambiente de ensino adequado à aprendizagem. Os descritores dessa dimensão analisam o impacto dos testbeds na aprendizagem do aluno, bem como reflete as adequações metodológicas para se trabalhar com esses ambientes em sala de aula. Na dimensão Avaliação são definidos os mecanismos de análise de aprendizado dos alunos e sua satisfação em relação à metodologia utilizada. O objetivo dos seus descritores é verificar se ocorreu aprendizagem e se os objetivos de curso definidos inicialmente foram atendidos. Por fim, a dimensão Design de Interface está relacionada, principalmente, à análise da interface do AVA e disponibilização do material didático na plataforma. Seu objetivo é verificar se o design do AVA e dos materiais didáticos facilitam o acesso às informações através de uma boa interação e navegabilidade.

\section{A experiência: curso de extensão SDN-IPS}

O curso de extensão "Sistemas de Detecção de Intrusão baseado em SDN", foi gratuito e oferecido pela Universidade Federal da Bahia (UFBA) Com participação de 21 alunos, ele foi realizado durante os meses de abril e maio de 2018, na modalidade semipresencial com carga horária total de 24 horas. O objetivo do curso foi apresentar conceitos e práticas relacionadas à construção de um Sistema de Prevenção de Intrusão baseado no paradigma de Redes Definidas por Software (do inglês, Software Defined Networking - SDN). 
A seleção dos alunos foi realizada com base em um conjunto de questões técnicas presentes no formulário de inscrição. As respostas a esse formulário guiaram a estruturação do plano de aulas, privilegiando discussões em temas cujos alunos apresentavam menor conhecimento. A ementa do curso incluiu os seguintes conteúdos: SDN e construção de ambientes experimentais, especialmente o testbed FIBRE; Roteamento na Internet através do protocolo BGP e do paradigma SDN; Sistema de Detecção e Prevenção de Intrusão (IPS); e técnicas de contenção de ataques cibernéticos.

As aulas presenciais ocorreram em um laboratório de informática da universidade, sendo os primeiros 40 minutos destinados à exposição teórica do assunto, que serviria como base para a realização da atividade prática subsequente. As práticas de laboratório desempenharam papel fundamental no processo de ensino e aprendizagem do aluno, tendo em vista que o aluno era estimulado a compreender os conceitos apresentados a partir de uma experiência próxima ao ambiente real. Em particular, as atividades práticas foram conduzidas a partir do ambiente testbed FIBRE. Tal ambiente, além de permitir a instalação e configuração das ferramentas e protocolos necessários ao software SDN-IPS, permitia ao aluno o uso de ferramentas de monitoramento e resolução de problemas similares àquelas utilizadas no cotidiano de profissionais da área.

Roteiros de laboratório eram fornecidos aos alunos, tal como desafios técnicos para serem resolvidos em casa. A possibilidade de realizar as atividades práticas em casa é uma das vantagens dos ambientes de testbeds, pois seu acesso está disponível $100 \%$ do tempo, bastando apenas um computador com conectividade à Internet. Com o intuito de auxiliar e mediar as atividades realizadas fora do espaço físico do curso, foi escolhido o LMS Moodle da universidade para hospedar o AVA. O Moodle foi essencial para disseminação do conteúdo teórico anterior à aula, de tal modo que o aluno pudesse previamente estudar e se familiarizar com os conceitos que seriam abordados nas aulas.

O curso foi dividido em quatro módulos, e ao final de cada módulo, o aluno respondia um questionário no modelo Quiz e realizava os roteiros das práticas de laboratório. Os questionários Quiz foram aplicados a fim de que o aluno fizesse uma auto avaliação sobre o seu progresso, oportunizando revisão específica de temas cujo desempenho no Quiz não fora satisfatório. Por outro lado, o roteiro de laboratório além de incentivar a aprendizagem através da prática, também contribuiu para o desenvolvimento de habilidades de resolução de problemas, visto que o testbed, ao se aproximar de ambiente real, também apresenta inconsistências e instabilidades tal qual equipamentos reais.

A experiência na execução do curso demonstrou que os estudantes foram expostos a novas experiências práticas que normalmente não são alcançadas em ambientes tradicionais de ensino de redes de computadores, comumente baseados em simuladores e emuladores. As atividades executadas em uma rede de experimentação baseada em equipamentos reais, proporcionaram maior entusiamo e identificação dos alunos, além de maior predisposição a participar ativamente do processo de aprendizagem. Tais resultados qualificam, portanto, a adoção dos testbeds para experiências de ensino na área de redes de computadores, expandindo seu uso original cujo foco esteve majoritariamente associado a execução de experimentos de pesquisa. 
IX Congresso Brasileiro de Informática na Educação (CBIE 2020)

Anais do XXXI Simpósio Brasileiro de Informática na Educação (SBIE 2020)

\section{Resultados}

A Figura 2 representa as etapas de construção do dispositivo RUBIK descrito na Seção 3. Já os resultados da sua aplicação são demonstrados nas Tabelas 1, 2, 3, 4 e 5.

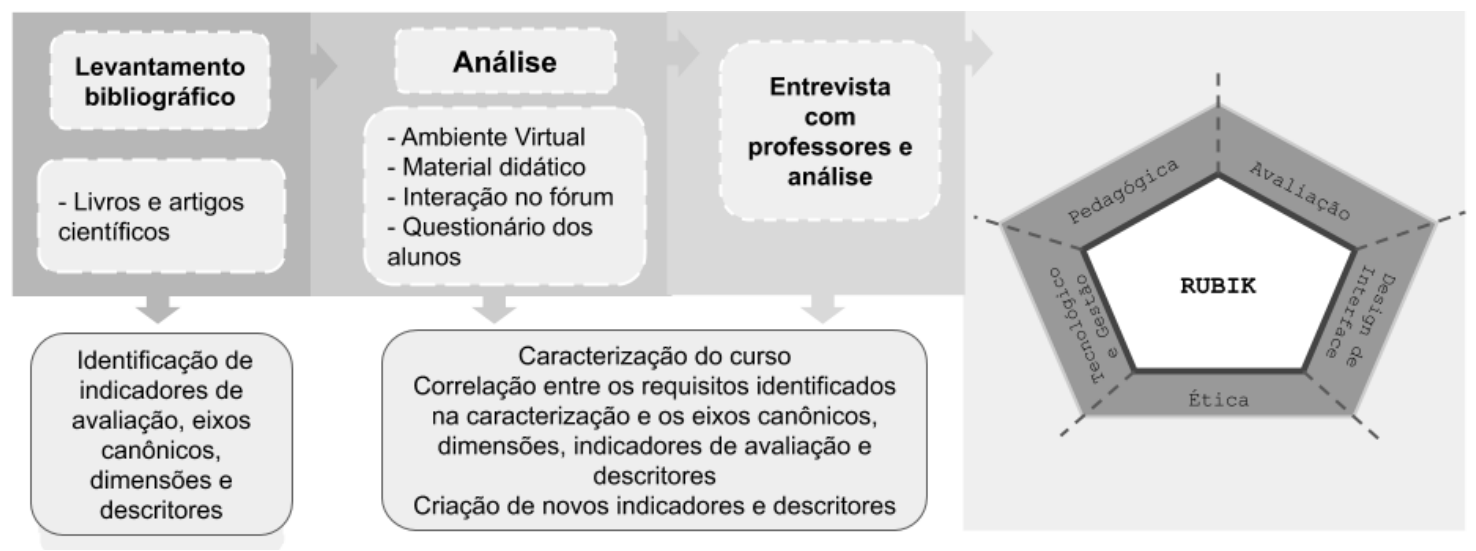

Figura 2. Etapas de Construção do Dispositivo de Avaliação RUBIK

Tabela 1. Dimensão Pedagógica

\begin{tabular}{|c|l|}
\hline Indicador de Avaliação & \multicolumn{1}{c|}{ Análise Realizada } \\
\hline $\begin{array}{c}\text { Processo de análise, desenvolvimento e } \\
\text { organização de conteúdo didático }\end{array}$ & $\begin{array}{l}\text { Conteúdo condizente com os objetivos do curso e com grau de dificuldade gradativo. Conteúdo teórico relacionado } \\
\text { aos experimentos práticos o que permite afirmar uma excelente integração entre atividade prática e conteúdo teórico. }\end{array}$ \\
\hline Ressignificação das práticas pedagógicas & $\begin{array}{l}\text { Aula deixou de ser apenas expositiva, para uma intercalação com a prática (uma atividade prática para cada conceito } \\
\text { teórico trabalhado). Conteúdo considerado relevante pois proporcionava possibilidades de aplicação nos ambientes de } \\
\text { trabalhos/estudos dos participantes. Professores motivados a criar estratégias de ensino que despertassem a } \\
\text { autonomia dos alunos. Baixo estimulo dos professores em promover convivio social nos AVA. }\end{array}$ \\
\hline Conscientização & $\begin{array}{l}\text { As posturas adotadas pelos professores estimularam o ensino centrado no aluno, permitindo que estes tivessem } \\
\text { controle e autonomia dos seus processo de ensino-aprendizagem. Professores se portaram como mediadores do } \\
\text { processo, abandonando a postura de detentor do conhecimento. }\end{array}$ \\
\hline Engajamento ativo & $\begin{array}{l}\text { Objetivos do curso alcançados, participantes satisfeitos com o conhecimento adquirido, garantindo a capacidade de } \\
\text { reaplicar esses conhecimentos em outros espaços. Professores mediadores, instruindo sempre que possivel a busca } \\
\text { por informações nos diversos materiais disponibilizados. Baixa participação e colaboração no fórum de discussão. }\end{array}$ \\
\hline
\end{tabular}

Na Tabela 1, tem-se a síntese dos resultados da dimensão Pedagógica. A análise demonstrou que os conteúdos selecionados, além de serem relevantes para a realidade dos alunos, apresentaram bom equilíbrio em relação aos conceitos teóricos e à prática. Além disso, a disponibilização prévia dos roteiros das práticas possibilitou que o aluno pudesse assumir uma postura mais autônoma. Ademais, a postura docente adotada também foi considerada adequada, pois estimulou o ensino centrado no aluno. Já o AVA foi pouco explorado no curso, o que acarretou em baixo compartilhamento de experiências entre os alunos. Para a produção de novos cursos parece ser importante ao professor dedicar mais atenção aos indicadores "ressignificação das práticas pedagógicas" e "engajamento ativo", visto que a própria experiência no testbed exige empenho por novas estratégias de ensino, principalmente no que se refere a iniciativas de interação e dinamismo.

Com os resultados obtidos na dimensão Ética, apresentados na Tabela 2, percebeuse a importância da boa relação aluno-professor para o ensino e a aprendizagem de qualidade. A boa relação entre aluno e professor ajuda a dirigir o processo educativo, tornando a aprendizagem mais ou menos facilitada, por isso, o professor deve possuir qualidades como empatia e apreço pelo aluno. Assim, parece ser importante que essa dimensão norteie a postura e o comportamento dos professores e alunos durante todo o curso. 
IX Congresso Brasileiro de Informática na Educação (CBIE 2020)

Anais do XXXI Simpósio Brasileiro de Informática na Educação (SBIE 2020)

Tabela 2. Dimensão Ética

\begin{tabular}{|c|c|}
\hline Indicador de Araliação & Análise Realizada \\
\hline Reeducação / modificação de perspectivas & Excelente relação professor-aluno e boa postura ético-profissional dos professores em conduzir o curso. \\
\hline
\end{tabular}

O resultado da aplicação na dimensão Tecnológica e de Gestão pode ser visto na Tabela 3. Nessa dimensão detectamos que o uso de testbed proporcionou um espaço de aprendizagem centrado no aluno, o que confirma seus benefícios para o ensino. Ainda nessa dimensão, entendeu-se que o AVA poderia ter contribuído melhor para a aprendizagem colaborativa, caso houvesse maior estímulo dos professores. Neste sentido,é importante destacar que para um experiência de ensino por meio de testbeds bem sucedida, os professores devem estimular a troca de interações entre os alunos de forma a produzir conhecimentos por meio das experiências individuais de cada um, assim, recomenda-se que o indicador "uso das tecnologias de informação e comunicação" seja revisto e melhor trabalhado na produção de novos cursos.

Tabela 3. Dimensão Tecnológica e de Gestão

\begin{tabular}{|c|l|}
\hline Indicador de Avaliação & \multicolumn{1}{c|}{ Análise Realizada } \\
\hline Inovação tecnológica & $\begin{array}{l}\text { Passagem de uma aprendizagem passivo/receptivo para ativo/questionador proporcionado devido as caracteristicas do } \\
\text { ambiente de testbed. Criação de diversas estratégias de ensino para contornar as fathas que ocorreram no ambiente de } \\
\text { testbed durante os experimentos. Ambiente de experimentação com baixo custo de implementação, o que possibilita } \\
\text { seu uso em sala de aula. }\end{array}$ \\
\hline $\begin{array}{c}\text { Uso das Tecnologias de Informação e } \\
\text { Comunicação - TIC }\end{array}$ & $\begin{array}{l}\text { Uso do AVA pouco explorado. Utilizado basicamente para promover trocas de informações entre os participantes } \\
\text { (avisos), não contribuindo para criação de um espaço virtual colaborativo. }\end{array}$ \\
\hline
\end{tabular}

A Tabela 4 apresenta os resultados obtidos na dimensão de Avaliação e demonstra que houve satisfação dos alunos com o curso, o que aponta que a experiência com uso de testbeds no ensino é interessante e enriquecedora do ponto de vista dos alunos. Ainda nessa dimensão foi aplicada a matriz de análise de conteúdo desenvolvida por [Lago 2013] para avaliar as interações no AVA. A aplicação da matriz demonstrou que a maior parte das mensagens trocadas não contribuiu para a aprendizagem, pois se tratavam de mensagens aleatórias; além disso, foi possível perceber razoável mediação dos professores, limitando-se a responder questionamentos. Para novos cursos, orienta-se que o professor desenvolva estratégias para fortalecer a comunicação nas ferramentas síncronas e assíncronas do AVA de forma a ampliar as possibilidade de aprendizagem.

Tabela 4. Dimensão Avaliação

\begin{tabular}{|c|l|}
\hline Indicador de Avaliação & \multicolumn{1}{c|}{ Análise Realizada } \\
\hline Processos avaliativos & Avaliação baseada na aplicação de questionário que considerava feddback do aluno para alguns aspectos do curso. \\
\hline Grau satisfatório dos participantes & Participantes satisfeitos com o curso. Professores organizados, seguindo fielmente todo o cronograma de atividades. \\
\hline Análise das interações no AVA & $\begin{array}{l}\text { Houve baixa troca de experiências pesscais entre os participantes. Mensagens aleatórias durantes as discussões, não } \\
\text { agregando conhecimento significativo. Razóivel mediação dos professores, limitando-se a esclarecer dúvidas e indicar } \\
\text { leituras, sem estimular a interação como forma de construção para novos conhecimentos. }\end{array}$ \\
\hline
\end{tabular}

Na dimensão Design de Interface, cujos resultados são apresentados na Tabela 5, foi percebido que o material didático disponibilizado, apesar de não dialógico, serviu como suporte para a realização de práticas por alguns alunos. Ainda nessa dimensão, destaca-se a necessidade de organização na disponibilização de conteúdo no espaço virtual, pois uma boa interação e navegabilidade facilitam o acesso à informação. No curso, 
IX Congresso Brasileiro de Informática na Educação (CBIE 2020)

Anais do XXXI Simpósio Brasileiro de Informática na Educação (SBIE 2020)

a personalização do AVA não seguiu algumas recomendações de acessibilidade e usabilidade. Assim, recomenda-se que em novos cursos haja atenção extra aos indicadores relacionados a essa dimensão, visto que o uso eficiente do AVA e um bom material didático podem incentivar a colaboração entre os alunos e facilitar o aprendizado.

Tabela 5. Dimensão Design de Interface

\begin{tabular}{|c|l|}
\hline Indicador de Avaliação & \multicolumn{1}{c|}{ Análise Realizada } \\
\hline Interface do AVA & AVA com informações dispersas e sem tópicos para organização de conteúdo, o que dificultava sua filtragem. \\
\hline Material didático interativo & $\begin{array}{l}\text { Adoção de material em diversos formatos (artigos, tutorial, imagens). Por vezes o material conseguiu suprir a ausência } \\
\text { fisica do professor, permitindo que sua leitura se tornasse suficiente para a resolução das atividades propostas. }\end{array}$ \\
\hline
\end{tabular}

Com a aplicação do dispositivo de avaliação RUBIK, foi possível avaliar a experiência do curso realizado, percebendo que os ambientes de testbeds são significativos e promissores para promover o ensino de redes, além de identificar oportunidades de ajustes no próprio dispositivo. Essa experiência pode contribuir ao nortear professores que desejam fazer a inclusão dessa ferramenta em suas aulas, mas não sabem quais indicadores adotar para avaliar seus resultados.

\section{Considerações finais}

Este artigo apresentou a experiência de um curso de extensão que utilizou testbeds como laboratório prático para realização de exercícios na área de redes de computadores. A avaliação através do RUBIK aconteceu após o fim do curso e analisou o uso dos testbeds como mecanismo de ensino. Através da sua aplicação, foi possível obter resultados que contribuem para viabilizar uma experiência de sucesso, garantindo cursos que possibilitem uma aprendizagem progressiva e centrada no aluno. Ainda como resultados, percebemos que a iniciativa de inserção dos testbeds como espaços de aprendizagem representa um ganho favorável para o ensino, proporcionando aos alunos a realização de experimentos em um cenário totalmente real, o que normalmente não é alcançado com os softwares simuladores/emuladores.

Além disso, as dimensões e indicadores definidos no RUBIK contribuem para oferecer aos professores um ponto de partida para produção de novos cursos, além de fornecer recursos que facilitem sua avaliação. Assim, os professores podem utilizar os resultados obtidos com o dispositivo RUBIK para melhorar suas experiências de ensino em redes de computadores, através do atendimento aos indicadores e descritores definidos no dispositivo, atentando-se principalmente para critérios como: postura docente, iniciativa, dinamismo e interação, considerados essenciais em uma experiência de ensino com testbeds. Ademais, os resultados sinalizam ainda, atitudes e postura inadequadas que podem resultar em uma prática de ensino frustrada, portanto, o acolhimento de tais recomendações podem resultar em melhores experiências de ensino.

Ainda que os resultados obtidos com o RUBIK demonstrem que o uso de testbeds para o ensino de redes é algo benéfico, é importante ressaltar que a avaliação deste trabalho está limitada a um único curso de extensão, e que por isso precisa ser expandida para outras modalidades de cursos que utilizem testbeds para realização de laboratórios práticos. Assim, a realização de experiências como essa são importantes não só para aumentar a qualidade dos cursos propostos, possibilitando identificar seus pontos 
positivos e limitações, mas também para a própria validação do RUBIK como dispositivo de avaliação. Como continuação deste trabalho almeja-se a atualização do RUBIK, tornando-o ainda mais específico para o contexto dos testbeds, bem como a avaliação de quais dimensões do dispositivo possuem maior influência no ensino e na aprendizagem. Para isso, realizaremos uma entrevista com professores de redes de computadores que possuem experiências com uso de testbeds, tanto para pesquisa quanto para o ensino.

\section{Agradecimentos}

Os autores agradecem o apoio do Conselho Nacional de Desenvolvimento Científico e Tecnológico (CNPq) e da Fundação de Amparo à Pesquisa do Estado da Bahia (FAPESB).

\section{Referências}

Berman, M., Chase, J. S., Landweber, L., Nakao, A., Ott, M., Raychaudhuri, D., and Ricci, Robert e Seskar, I. (2014). Geni: A federated testbed for innovative network experiments. Computer Networks, 61:5-23.

Ciuffo, L., Salmito, Tiago Rezende, J., and Machado, I. (2016). Testbed fibre: Passado, presente e perspectivas. In Anais do WPEIF 2016 Workshop de Pesquisa Experimental da Internet do Futuro, pages 3-6. sn.

Crivelaro, L. P. (2014). Indicadores que levam um professor a tornar-se colaborativo. $\mathrm{PhD}$ thesis, Unicamp, Campinas, SP.

Dias, G., de Rezende, J., Ciuffo, L., Machado, I., Silva, F., Carvalho, C., Redígolo, F., Martins, J., Sampaio, L., and Gomes, J. (2019). Sfi2 - slicing future internet infrastructures project.

Khan, B. H. (2001). A framework for web-based learning. Web-based training, pages 75-98.

Lago, A. F. (2013). Comunidades e interatividade: um estudo sobre cursos on-line como espaço de (in) formação.

Mikroyannidis, A., Collins, D., Tranoris, C., Denazis, S., Pareit, D., Gerwen, J.-V., Moerman, I., Jourjon, G., Fourmaux, O., Domingue, J., et al. (2017). Forge: an elearning framework for remote laboratory experimentation on fire testbed infrastructure. In Building the Future Internet through FIRE-2016 FIRE Book: A Research and Experiment based Approach/Serrano, M.[edit.]; et al.[edit.], pages 521-559.

Pedroso, D., Lopes, B., Marcondes, C., and Barea, E. (2018). Simulação de ambientes de redes utilizando a testbed fibre - aplicações na pesquisa e no ensino. In Anais do III Workshop do Testbed FIBRE, Porto Alegre, RS, Brasil. SBC.

Silva, A. M. C. and Silva, B. D. a. (2008). Dispositivos de avaliação de projectos em tic: uma experiência em portugal. Revista Diálogo Educacional, 8(24):389-404.

Tavares, T. N. (2018). Ctc-proposta de uma arquitetura para testbed universitária. Revista Eletrônica de Iniciação Científica em Computação, 16(2).

Valenzuela, G. and Amaral, L. (2018). Laboratório de roteamento bgp para ambiente educacional no testbed fibre. In $3^{\circ}$ Workshop do testbed FIBRE: uso de Plataformas Experimentais no auxílio do aprendizado de Redes de Computadores (FIBRE), volume 3. SBC. 